\title{
Molecular Tools for Staphylococcus aureus Typing: Basic and Clinical Aspects
}

\author{
Kanokporn Mongkolrattanothai, MD
}

Advances in molecular techniques for bacterial strain typing have enhanced our understanding of disease epidemiology and provided insight into the evolution of bacterial pathogens. A number of strain typing methods have been developed to explore the genotypes of Staphylococcus aureus, one of the most common pathogenic organisms responsible for a wide variety of infectious syndromes. However, each method has its own advantages and disadvantages, so the optimal method of bacterial strain typing depends on the objectives of data collection and available resources ${ }^{1}$. For instance, if the goal is to characterize a local or outbreak investigation, the techniques with high discriminatory power (an ability to distinguish between epidemiologically-unrelated bacterial strains) should be chosen. However, bacteria will inevitably accumulate mutations, an essential mechanism that plays a central role in bacterial evolution, in their genomes over time. As a result, if the goal is to investigate at a global level or monitor the long-term evolution of bacteria, the sequence-based techniques that analyze more stable genetic markers, such as housekeeping genes, should be used in order to recognize ancestral relationships among bacterial strains. Here, we summarize some of the key tools for $S$. aureus typing, and their applications for healthcare providers.

In epidemiological studies, pulsed-field gel electrophoresis (PFGE) has been a valuable tool to characterize $S$. aureus isolates for the past few decades. PFGE is a technique based on the digestion of bacterial DNA with Smal restriction endonuclease, thereby generating large fragments of DNA that are separated by pulsed-field electric fields. A national PFGE-based typing system for $S$. aureus, designated as pulsed-

$$
\begin{array}{r}
\begin{array}{r}
\text { Corresponding author: } \\
\text { Kanokporn Mongkol- } \\
\text { rattanothai, MD }
\end{array} \\
\begin{array}{r}
\text { Contact Information: } \\
\text { kanokporn.mongkolrat } \\
\text { tanothai@ttuhsc.edu }
\end{array} \\
\begin{array}{r}
\text { DOI: } 10.12746 / \text { swrccc2013.0102.014 }
\end{array}
\end{array}
$$

field types USA100 through USA1200, has been established by the Centers for Disease Control and Prevention, and has served as an important tool to facilitate the exchange of PFGE strain typing data and epidemiologic information among reference laboratories ${ }^{2}$. PFGE has good discriminatory power and is suitable for short-term epidemiology, but it is laborious, time consuming, and can be performed only in well-equipped laboratories. In order to avoid the arduous process, polymerase chain reaction (PCR)based typing methods have been developed and are commonly used due to their simplicity and ability to provide rapid results. PCR is the molecular technology that is based on amplification of particular genetic targets. PCR-based methodologies allow generation of DNA profiles that can be analyzed using techniques such as gel electrophoresis or DNA sequence analysis. In gel electrophoresis, amplified PCR products are separated to yield a DNA band pattern, aka DNA fingerprint, which is characteristic of a particular bacterial strain. Multiple-locus variable number of tandem repeat fingerprinting (MLVF), a PCR-based method, is one of the highly discriminatory fingerprinting assays for $S$. aureus typing ${ }^{3,4}$. MLVF targets the amplification of the five (sdr, clfA, clfB, sspA, and spa) tandem repeat loci, followed by the separation of amplified products by gel electrophoresis. MLVF is a simple, cheap, and rapid method for fingerprinting analysis.

DNA sequence-based typing methods are the most powerful tool for bacterial strain typing because of their accuracy, reproducibility, and exchangeability of the results. However, their discriminatory power depends on the number, degree of variability, and stability of loci that are selected for sequencing. spa typing is a single-locus typing based on sequencing of the polymorphic $X$ region of the protein A gene (spa) of $S$. aureus bacteria ${ }^{5}$. Isolates are assigned to particular spa types using the spa typing website (http://www. spaserver.ridom.de). Several studies have demon- 
strated that spa typing is highly discriminatory, and useful in both local and global epidemiological studies ${ }^{6}$. Multilocus sequence typing (MLST) is another typing system that is based on nucleotide sequences of seven housekeeping genes of $S$. aureus (arcc, aroe, glpf, gmk, pta, tpi, yqil) ${ }^{7}$. The sequences at each of the seven loci are compared with the known alleles by use of the $S$. aureus MLST database (http://www. mlst.net), and are assigned the allele number. The alleles of the seven loci define the allelic profile and sequence type (ST) of each isolate. Since mutations accumulate slowly in housekeeping genes, the MLST scheme is used to delineate clusters of closely-related strains. It has been an excellent tool for phylogenetic and evolutionary analyses. However, MLST is not suitable to characterize the differences in strains within an outbreak as its power to resolve small evolutionary differences is too low. In addition, the costs of sequencing currently limit their routine uses for most epidemiological studies.

To better understand the clinical utility of each typing method, clinical scenarios follow as examples.

\section{Clinical scenario 1:}

A clinician on a neonatal intensive care unit (ICU) notified the Infection Control Division that there were three hospitalized patients who developed nosocomial-onset MRSA infections within the past two weeks. She was concerned that this could be an outbreak of MRSA in the unit. To investigate for a possible an MRSA outbreak, antimicrobial susceptibility patterns of those three MRSA isolates were reviewed and found to be identical. As a pressing need to control a potential MRSA outbreak, surveillance cultures were obtained from all newborn infants in the unit. Nine other infants were found to have MRSA colonization. All health care workers who had direct patient contact were also screened for MRSA colonization. All MRSA isolates were analyzed by MLVF and PFGE to determine their genetic relatedness. These two methods, as well as spa typing, are appropriate to determine genetic relatedness among MRSA isolates in a potential outbreak investigation. The MLVF patterns of MRSA isolates from all newborn infants, except that from infant 9 , and from healthcare worker
12 and 15 were all identical (Figure 1). These findings, which were consistent with PFGE results (data not shown), are suggestive of an MRSA outbreak in this neonatal ICU.

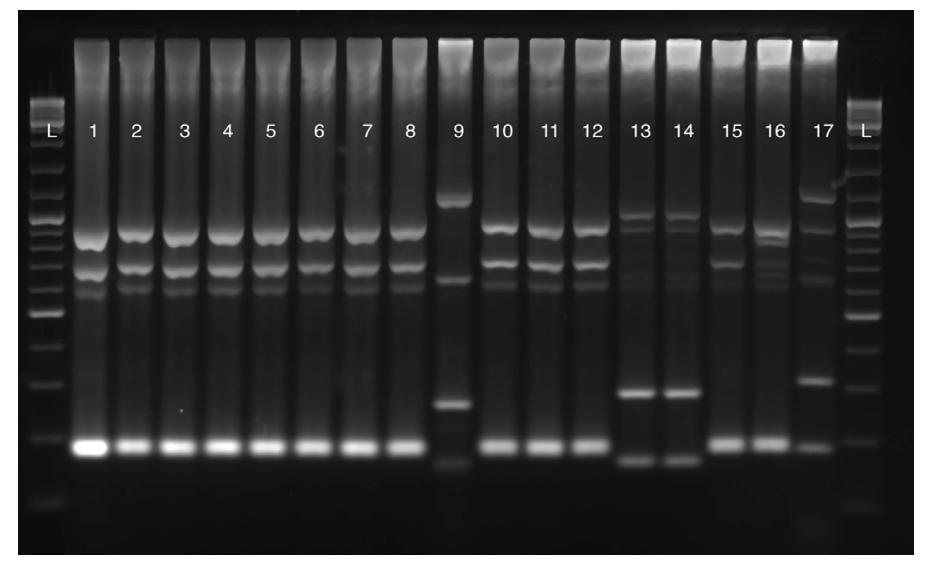

Figure 1. MLVF analysis of MRSA isolates in an outbreak investigation in a neonatal ICU. L, molecular weight marker; 1-11, MRSA isolates from infants; 12-17, MRSA isolates from healthcare workers.

\section{Clinical scenario 2:}

A study to characterize the epidemiology of MRSA isolates in an ICU during a 10-year-period (2002-2011) was conducted. The study sought to determine the dominant clones of MRSA circulating in that ICU, and understand the molecular evolution of MRSA within the unit. In this setting, MLST or spa typing, or both as was done in this case, are the preferred methods of bacterial typing to distinguish MRSA clones. A total of 450 MRSA non-duplicate isolates collected during the study period were analyzed. The results revealed that MRSA isolates were classified into nine spa types and six STs. During the years from 2002-2007, the predominant MRSA clone was ST5-MRSA-spa t003. However, since 2007, ST5-MRSA-spa t002 rapidly replaced t003 and became the major clone. Another clone, ST8-MRSA-spa t008, the most common clone of community-associated MRSA (CA-MRSA) in the US, emerged in 2005 and constantly existed in the ICU with the highest incidence rate at 35\% in year 2011. The increase in the hospital onset of CA-MRSA infection rate is suggestive of the migration of CA- 
MRSA strains to the hospital environments. Of note, this scenario is based on simulated data.

\section{Key Statements}

1. The techniques with high discriminatory power should be chosen if the goal is to characterize a local or outbreak investigation.

2. If the goal is to investigate at a global level or monitor the long-term evolution of bacteria, the techniques that analyze stable genetic markers should be used to recognize ancestral relationships among strains.

KEY wORDS- Staphylococcus aureus, molecular typing.

Author Affiliation: Dr Mongkolrattanothai is an infectious disease subspecialist in Department of Pediatrics, TTUHSC.

Received: $12 / 23 / 2012$

Accepted: $3 / 1 / 2013$

Reviewers: Jane Colmer-Hamood, PhD, David Straus PhD, Ted Reid, PhD

Published electronically: 4/15/2013

Conflict of Interest Disclosures: None phoresis types. J Hosp Infect, 2009. 71(4): p. 333-9.

5. Shopsin, B., et al., Evaluation of protein A gene polymorphic region DNA sequencing for typing of Staphylococcus aureus strains. J Clin Microbiol, 1999. 37(11): p. 3556-63.

6. Koreen, L., et al., spa typing method for discriminating among Staphylococcus aureus isolates: implications for use of a single marker to detect genetic micro- and macrovariation. J Clin Microbiol, 2004. 42(2): p. 792-9.

7. Robinson, D.A. and M.C. Enright, Multilocus sequence typing and the evolution of methicillin-resistant Staphylococcus aureus. Clin Microbiol Infect, 2004. 10(2): p. 92-7.

\section{REFERENCES}

1. Shopsin, B. and B.N. Kreiswirth, Molecular epidemiology of methicillin-resistant Staphylococcus aureus. Emerg Infect Dis, 2001. 7(2): p. 323-6.

2. McDougal, L.K., et al., Pulsed-field gel electrophoresis typing of oxacillin-resistant Staphylococcus aureus isolates from the United States: establishing a national database. J Clin Microbiol, 2003. 41(11): p. 5113-20.

3. Karynski, M., et al., Molecular surveillance of methicillin-resistant Staphylococcus aureus by multiple-locus variable number tandem repeat fingerprinting (formerly multiple-locus variable number tandem repeat analysis) and spa typing in a hierarchic approach. Diagn Microbiol Infect Dis, 2008. 62(3): p. 255-62.

4. Moser, S.A., et al., Multiple-locus variable-number tandem-repeat analysis of meticillin-resistant Staphylococcus aureus discriminates within USA pulsed-field gel electro- 